\title{
CRNDE: An important oncogenic long non-coding RNA in human cancers
}

\author{
Jiaming Zhang ${ }^{1,2}$ (D) | Minuo Yin ${ }^{3} \mid$ Gang Peng $^{1} \mid$ Yingchao Zhao $^{1}$
}

\author{
${ }^{1}$ Cancer Center, Union Hospital, Tongji \\ Medical College, Huazhong University of \\ Science and Technology, Wuhan, China \\ ${ }^{2}$ Department of Orthopedics, Tongji \\ Hospital, Tongji Medical College, Huazhong \\ University of Science and Technology, Wuhan, \\ China \\ ${ }^{3}$ Department of Obstetrics and \\ Gynecology, Union Hospital, Tongji Medical \\ College, Huazhong University of Science and \\ Technology, Wuhan, China

\section{Correspondence} \\ Yingchao Zhao and Gang Peng, Cancer \\ Center, Union Hospital, Tongji Medical \\ College, Huazhong University of Science and \\ Technology, Wuhan, China. \\ Emails: 2006xh0836@hust.edu.cn and \\ penggang1977@hotmail.com \\ Funding information \\ National Natural Science Foundation of China, \\ Grant/Award Number: No. 81502657
}

\begin{abstract}
Aberrant overexpression of long non-coding RNA CRNDE (Colorectal Neoplasia Differentially Expressed) is confirmed in various human cancers, which is correlated with advanced clinicopathological features and poor prognosis. CRNDE promotes cancer cell proliferation, migration and invasion, and suppresses apoptosis in complicated mechanisms, which result in the initialization and development of human cancers. In this review, we provide an overview of the oncogenic role and potential clinical applications of CRNDE.
\end{abstract}

\section{1 | INTRODUCTION}

Long non-coding RNAs (IncRNAs) are broadly defined as a class of non-coding transcripts more than 200 nucleotides in length, which lack an open reading frame. ${ }^{1-3}$ Regardless of the fact that precise roles of the vast majority of $\sim 40000$ IncRNAs are still under investigation, ${ }^{4}$ IncRNAs are shown to be potential key regulators in the processes of proliferation, migration and invasion of cancer cells $\mathrm{s}^{5-8}$ by regulating gene expression patterns in various levels including the chromatin-organizational, transcriptional and post-transcriptional regulation. $^{9-13}$

Colorectal neoplasia differentially expressed (CRNDE), first reported to be upregulated in colorectal adenomas and carcinomas, is a gene locus hCG_1815491 on chromosome 16 located on the strand opposite to the adjacent IRX5 gene. ${ }^{14,15}$ Due to its transcripts with low coding potentials, CRNDE is classified as a long non-coding RNA. ${ }^{14,15}$ Interestingly, CRNDE displays temporal and tissue-specific expression patterns. It is faintly expressed in normal tissues including white blood cells and colorectal mucosa, while highly expressed in breast, testis, bronchial epithelium and parotid gland. ${ }^{15}$

In addition, CRNDE is elevated in various cancers including colorectal cancer (CRC), ${ }^{14,16-21}$ glioma, ${ }^{22-25}$ hepatocellular carcinoma ${ }^{26-28}$ and lung cancer, ${ }^{29}$ suggesting its irreplaceable roles in the initialization and development of human cancers. Due to alternative splicing, CRNDE derives at least 10 alternative RNA transcripts, among which CRNDE-a, -b, - d, -e, -f, - $h$ and -j are the major transcripts in cancer tissues and cell lines. ${ }^{14}$ In general, the known splice variants of CRNDE are upregulated in both neoplastic colorectal tissues and cell lines. However, it is an exception that CRNDE-d has a decreasing trend in neoplastic colorectal tissues, which is opposed in CRC cell lines HT29, $\mathrm{CaCo} 2$ and HCT116. ${ }^{14}$

Accumulating evidence has indicated the functions of CRNDE in regulation of cancer cell proliferation, migration, invasion and apoptosis. ${ }^{20,21,30-32}$ Furthermore, CRNDE was shown to be not only a diagnostic biomarker exhibiting high sensitivity and specificity in tissues and plasma, ${ }^{14,16,33}$ but also a prognostic 
predictor associated with clinicopathological features and outcomes in various cancers. The present review summarized current evidence regarding the abnormal expression, molecular mechanisms and clinical significance of CRNDE in human cancers (Tables 1 and 2).

\section{2 | CRNDE IN HUMAN CANCERS}

\section{1 | Colorectal cancer}

Many studies have demonstrated CRNDE as a remarkably upregulated IncRNA in CRC tissues and cell lines. ${ }^{14,16-21}$ Exosomal CRNDE derived from tumour cells, which was detectable and stable, was also upregulated in the serum of CRC patients. ${ }^{33,34}$ As an ideal prognostic predictor in colorectal cancer tissues and serum, elevated CRNDE was significantly correlated with large tumour size, ${ }^{16,17}$ regional lymph node metastasis, ${ }^{16,33,34}$ distant metastasis, ${ }^{16,33,34}$ advanced pathological stage ${ }^{17,20,34}$ and unfavourable outcomes. ${ }^{16,20}$ Due to its potential high specificity and sensitivity for colorectal adenomas and cancer, CRNDE also served as a promising biomarker in tissues and plasma. ${ }^{14,16,33}$

Involved in multiple cancer-related pathways, CRNDE plays important roles in the initialization and development of CRC. Ellis et al. ${ }^{35}$ demonstrated the mechanisms of CRNDE in the regulation of cellular metabolism in which cancer cells switch to aerobic glycolysis (Warburg effect). The repression of CRNDE by insulin and insulin-like growth factors (IGF) was eliminated by the inhibitors against either the PI3K/Akt/mTOR pathway or Raf/MAPK pathway, suggesting that CRNDE was a downstream target of both signalling cascades. Liu et al. ${ }^{16,33}$ found that the expression of CRNDE-h was positively correlated with IRX5 in CRC tissues. Ding et al. ${ }^{17}$ reported that the knockdown of CRNDE suppressed proliferation and induced apoptosis of CRC cells both in vitro and in vivo. Furthermore, CRNDE could epigenetically suppress the expressions of P21 and DUSP5 by recruiting EZH2, thus promoting CRC development. Jiang et al. ${ }^{20}$ demonstrated that CRNDE could form a functional complex with hnRNPUL2 to increase its stability and direct the transport of hnRNPUL2 between the nucleus and cytoplasm, which resulted in accelerated cell proliferation and migration by activating Ras/ MAPK signalling pathways.

CRNDE functions as a competing endogenous RNA to promote oncogenesis. Gao et al. ${ }^{18}$ confirmed that CRNDE promoted metastasis and oxaliplatin resistance by sponging miR-136 and derepressing its target, E2F1. Han et al. ${ }^{19}$ found CRNDE inhibited miR-181a-5p which targeted to beta-catenin and TCF4, and suppressed Wnt/beta-catenin signalling to promote colorectal cancer cell proliferation and chemoresistance. Yu et al. ${ }^{34}$ revealed that CRNDE was negatively correlated with miR-217 in colorectal cancer tissues and cell lines, and served as a competing endogenous RNA (ceRNA) through binding miR-217, which results in increasing the expression of TCF7L2 to activate Wnt/ beta-catenin signalling and promote cell proliferation, migration and invasion of CRC cells. ${ }^{21}$

\section{2 | Glioma}

The oncogenic role of CRNDE in glioma is another research hotspot. Zhang et al. ${ }^{22}$ analysed the IncRNA expression patterns in a set of gene expression profiles of 268 glioma specimens, and identified 127 differentially expressed IncRNAs, among which CRNDE were found to be highly upregulated in glioma (fold change $=32.0$ ). These are consistent with the profile results of Murat et al., ${ }^{23} \mathrm{Grzmil}$ et al. ${ }^{24}$ and Chen et al. ${ }^{25}$ The upregulation of CRNDE was confirmed in both glioma tissues and cell lines, ${ }^{30,36-38}$ which acted as an independent prognostic factor correlated with larger tumour size, ${ }^{37}$ higher grade, ${ }^{37}$ recurrence ${ }^{37}$ and poorer overall survival. ${ }^{37,39}$ Furthermore, the value of different transcript variants of CRNDE in clinical prognostication was also demonstrated and a low ratio of CRNDE-h/CRNDE-P predicted a more favourable disease outcomes. ${ }^{39}$

Multiple studies have tried to elucidate the mechanisms in which CRNDE promotes glioma. Based on bioinformatics analyses, Zhang et al. ${ }^{40}$ predicted multiple transcription factors binding to the promoter regions of CRNDE including c-Myc, TAF1, E2F6 and SMAD, which might participate in the dysregulation of CRNDE. Wang et al. $^{36}$ reported that histone acetylation in the promoter region might account for the elevated expression of CRNDE, and CRNDE could regulate mTOR signalling by phosphorylation of P70S6K in glioma. As a miRNA sponger, CRNDE promoted the malignant biological characteristics of glioma stem cells by negatively regulating miR-186, which targets to XIAP and PAK7, and regulating their downstream proteins including MARK2, cyclin D1, BAD and caspase $3{ }^{38}$ Furthermore, CRNDE also regulated the expression of miR-384 and its target PIWIL4, whose downstream proteins such as STAT3, cyclin D1, VEGFA, SLUG, MMP-9, caspase 3, Bcl-2 and Bcl-XI, play important roles in malignant biological characteristics of glioma cells. ${ }^{31}$ Kiang et al. ${ }^{30}$ confirmed that the expression of CRNDE was positively correlated with EGFR activation by a microarray data analysis. Li et al. ${ }^{41}$ found that the differentially expressed genes in CRNDE-overexpressed human astrocyte cell were involved in tolllike receptor (TLR) pathway, and further confirmed that CRNDE activated TLR3-mediated MyD88-independent TLR signalling pathway through TICAM1, PELI1 and RIPK2, and subsequently activated NF- $\kappa B$ and several cytokines, which finally resulted in tumourigenesis and tumour development.

\section{3 | Hepatocellular carcinoma}

The expression of CRNDE was significantly increased in human hepatic carcinoma (HCC) tissues and cell lines, ${ }^{26,27}$ the former of which was consistent with the previous microarray analysis results of Jin et al. ${ }^{28}$ who identified CRNDE as the top 20 upregulated IncRNA in 3 datasets of gene expression profiles of 117 HCC patients. Esposti et al. ${ }^{42}$ performed RNA-Seq in 10 HCC tissues to identify and characterize differentially expressed IncRNAs, among which CRNDE was identified as one of highly upregulated IncRNA with the fold change of 1.54. Moreover, CRNDE might serve as a potential diagnostic biomarker in $\mathrm{HCC}^{26}$ CRNDE was important for hepatoblastoma cell 


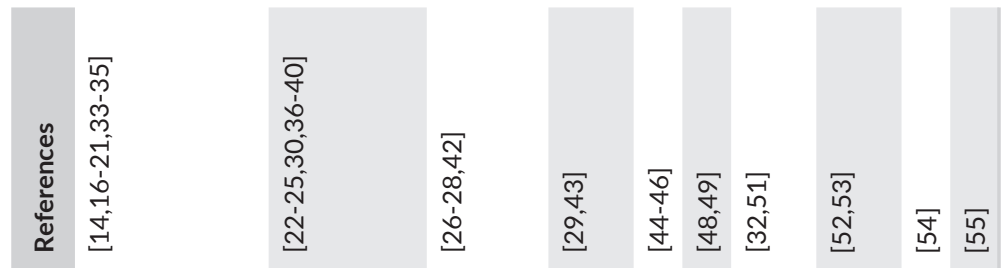

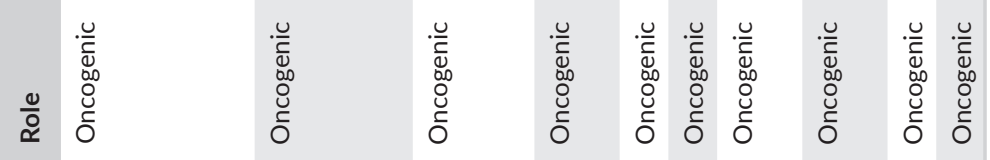

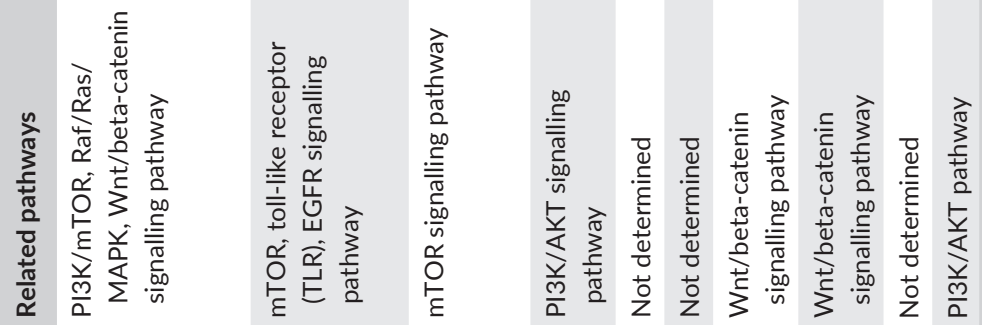

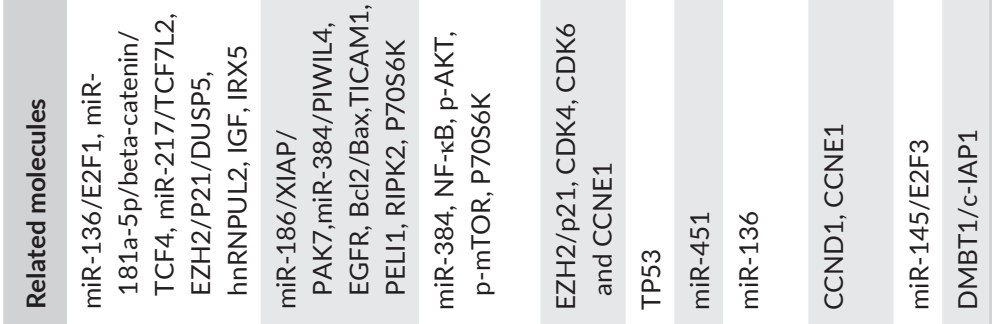

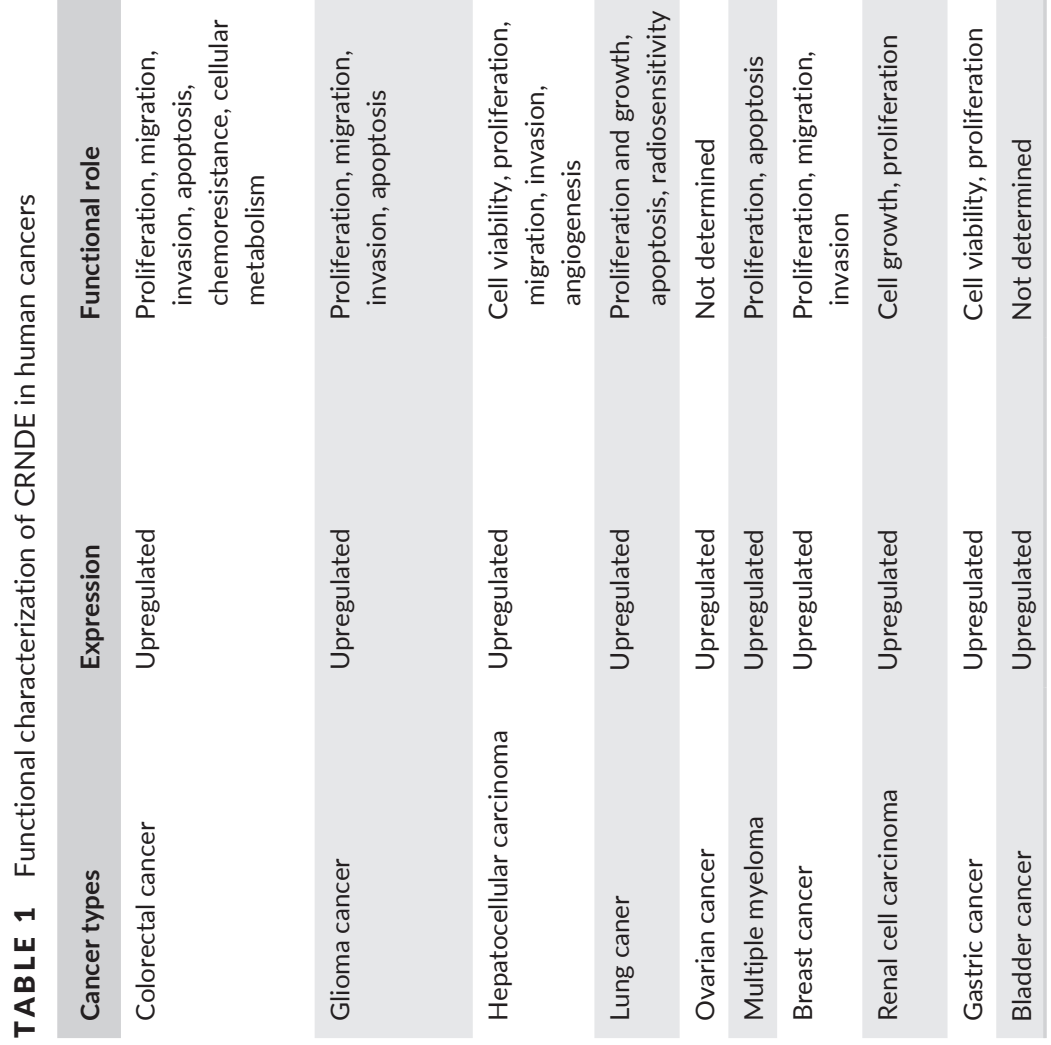




\begin{tabular}{|c|c|c|}
\hline Cancer types & Related clinical parameters & References \\
\hline Colorectal cancer & $\begin{array}{l}\text { Tumour size, lymph node metastasis, distant } \\
\text { metastasis, TNM stage, unfavourable survival }\end{array}$ & {$[16,17,20,33,34]$} \\
\hline Glioma cancer & $\begin{array}{l}\text { Tumour size, grade, recurrence, unfavourable } \\
\text { survival }\end{array}$ & {$[37,39]$} \\
\hline Lung caner & $\begin{array}{l}\text { Differentiation, lymph node metastasis, TNM } \\
\text { stage, radiotherapy response, unfavourable } \\
\text { survival }\end{array}$ & {$[29,43]$} \\
\hline ]Ovarian cancer & Recurrence, unfavourable survival & {$[45]$} \\
\hline Cervical cancer & $\begin{array}{l}\text { Invasion depth, lymph node metastasis, FIGO } \\
\text { stage, unfavourable survival }\end{array}$ & {$[47]$} \\
\hline Multiple myeloma & Unfavourable survival & {$[49]$} \\
\hline Breast cancer & Tumour size, TNM stage, unfavourable survival & {$[32]$} \\
\hline Papillary thyroid cancer & Gender, unfavourable survival & [57] \\
\hline
\end{tabular}

viability, proliferation and angiogenic effect in vitro as well as tumour growth and angiogenesis in vivo. ${ }^{27}$ In mechanism, CRNDE suppressed miR-384 and regulated NF- $\mathrm{KB}$ and $\mathrm{p}-\mathrm{AKT}$ expression to promote hepatic carcinoma cell proliferation, migration and invasion. ${ }^{26}$ CRNDE activated mTOR signalling by regulating the phosphorylation level of mTOR and P70S6K, which resulted in promoted hepatoblastoma cell functions and angiogenic effect. ${ }^{27}$

\section{4 | Lung caner}

Zhang et al. ${ }^{29}$ identified that CRNDE was significantly upregulated in lung adenocarcinoma (LAD) tissues and radio-resistant LAD cell lines, and was significantly correlated with poor differentiation, TNM stage and lymph node metastasis, radiotherapy response and shorter overall survival. CRNDE could interact with PRC2 and recruit its core component EZH2 to the promoter regions of p21 and repress the transcription of $\mathrm{p} 21$, which partly resulted in the radiosensitivity of LAD cells by affecting the G1/S transition and causing apoptosis. ${ }^{29}$ Liu et al. found the expression of CRNDE was significantly upregulated in nonsmall cell lung carcinoma (NSCLC) tissues and cell lines, and promoted NSCLC cell proliferation and growth through activating PI3K/AKT signalling. ${ }^{43}$ In addition, CRNDE modulated the expressions of CDK4, CDK6 and CCNE1, which were contributed to the cell cycle transition from G0/G1 stage to S stage. ${ }^{43}$

\subsection{Ovarian and cervical cancer}

Fifty-four differentially expressed IncRNAs were identified in ovarian cancer-associated fibroblasts compared to normal ovarian fibroblasts, and 16 IncRNAs were differentially expressed based on survival, included MALAT1, MEG3, TUG1, XIST and CRNDE. ${ }^{44}$ Szafron et al. ${ }^{45}$ found that 2 elevated CRNDE transcripts (FJ466685 and FJ466686, published in GenBank 2008) negatively influenced prognosis by significantly increasing risk of death and/or recurrence on 135 ovarian carcinomas patients with platinum compounds and either cyclophosphamide ( $P C, n=32$ ) or taxanes (TP, $n=103$ ), and the decreased expression of CRNDE was correlated with the accumulation of TP53. ${ }^{46}$
Han et al. found that CRNDE expression was significantly upregulated in 87 cervical cancer tissues, which was significantly correlated with the depth of cervical invasion, lymph node metastasis and FIGO stage, and predicted poorer overall survival. ${ }^{47}$

\section{6 | Haematologic malignancies}

Du et al. ${ }^{48}$ found that 5 of 9 differentially expressed IncRNAs, GAS5, CRNDE, LOC400657, PMS2P3 and TCL0000445, were associated with inferior prognosis and corresponded with 70 genes with $30 \%$ mapped to chromosome 1 by a IncRNA analysis based on microarrays of multiple myeloma. Meng et al. ${ }^{49}$ illustrated that CRNDE was upregulated in multiple myeloma samples and cell lines, which was closely related to tumour progression and poor survival. CRNDE induced the proliferation and antiapoptosis capability of multiple myeloma by acting as a ceRNA via negatively targeting miR-451. Subhash et al. ${ }^{50}$ identified 5800 hypermethylated and 12570 hypomethylated chronic lymphocytic leukaemia (CLL)-specific differentially methylated genes, among which hypermethylated CRNDE was validated and correlated with poor outcomes.

\section{7 | Breast cancer}

Maguire et al. ${ }^{51}$ found that differentially spliced events expressed in SF3B1 mutant tumours including CRNDE, ICA1, OBSL1, RPL31 and TMEM14C might constitute drivers of ER-positive breast cancers. Huan et al. ${ }^{32}$ found that CRNDE was remarkably upregulated in breast cancer tissues and cell lines, which was greatly associated with tumour size, TNM stage and unfavourable prognosis. CRNDE activated excessively $\mathrm{Wnt} /$ beta-catenin signalling pathway through repressing miR-136 expression.

\section{8 | Other cancers}

Shao et al. ${ }^{52}$ identified CRNDE as the highly expressed gene in renal cell carcinoma (RCC) patients by published microarray analyses, which was subsequently confirmed in 15 RCC tissues and 2 cell lines. CRNDE 
promoted cell proliferation through modulating the expression of CCND1 and CCNE1, and activating Wnt/beta-catenin signalling in RCC. Yang et al. ${ }^{53}$ found that CRNDE was highly expressed in clear cell renal cell carcinoma (ccRCC) and metastatic ccRCC samples, which suggested that CRNDE was important in the progression of ccRCC. Hu et al. ${ }^{54}$ showed that CRNDE acted as ceRNA to promote proliferation of gastric cancer cells by competitive sponging miR-145. Shen et al. ${ }^{55}$ authenticated that CRNDE acted as a scaffold to recruit the DMBT1 and c-IAP1, resulting in promoted PI3K/AKT pathway in bladder cancer. Lili et al. ${ }^{56}$ reported that CRNDE was upregulated in pancreatic cancer samples with a significant fold change of 1.77. A total of 734 IncRNAs were detected to be aberrantly expressed in a comprehensive study performed to screen IncRNA expression profiling with 507 papillary thyroid cancer (PTC) patients from The Cancer Genome Atlas RNA-sequencing datasets, among which CRNDE served as an ideal diagnostic factor remarkedly related to the progression and survival of PTC. ${ }^{57}$

\section{3 | CONCLUDING REMARKS AND FUTURE PERSPECTIVES}

As a newly characterized oncogene, CRNDE is an important IncRNA in human cancers. Accumulating evidence has confirmed the aberrant overexpression of CRNDE in various human malignancies, which is correlated with advanced clinicopathological features and poor prognosis. CRNDE promotes cancer cell proliferation, migration and invasion, and suppresses apoptosis in complicated mechanisms including competitively sponging tumour-suppressive microRNAs and recruiting chromatic modifiers. Nevertheless, the detailed regulatory mechanisms of CRNDE remain to be elucidated. In addition, efforts should be put forth to investigate the different functions of various alternative transcripts, and the lucid regulation to alternative splicing of CRNDE. In terms of clinical applications, CRNDE may serve as a potential biomarker for diagnosis and prognosis in various cancers. However, large-scale multi-centre cohort studies are expected to prove the clinical significance and practicability of CRNDE in future.

\section{ACKNOWLEDGEMENTS}

This study was supported by National Natural Science Foundation of China (Grant No. 81502657).

\section{CONFLICTS OF INTEREST}

The authors declared no conflict of interests about the publication of this paper.

\section{AUTHORS' CONTRIBUTIONS}

Conception and literature search: Jiaming Zhang. Manuscript writing and final approval: Jiaming Zhang, Minuo Yin, Gang Peng and Yingchao Zhao.

\section{ORCID}

Jiaming Zhang (iD http://orcid.org/0000-0001-8520-566X

\section{REFERENCES}

1. Maruyama R, Suzuki H. Long noncoding RNA involvement in cancer. BMB Rep. 2012;45:604-611.

2. Lian Y, Ding J, Zhang Z, et al. The long noncoding RNA HOXA transcript at the distal tip promotes colorectal cancer growth partially via silencing of $\mathrm{p} 21$ expression. Tumour Biol. 2016;37:7431-7440.

3. Li F, Cao L, Hang D, Wang F, Wang Q. Long non-coding RNA HOTTIP is up-regulated and associated with poor prognosis in patients with osteosarcoma. Int J Clin Exp Pathol. 2015;8:11414-11420.

4. Schlosser K, Hanson J, Villeneuve PJ, et al. Assessment of circulating LncRNAs under physiologic and pathologic conditions in humans reveals potential limitations as biomarkers. Sci Rep. 2016;6:36596.

5. Yang G, Lu X, Yuan L. LncRNA: a link between RNA and cancer. Biochem Biophys Acta. 2014;1839:1097-1109.

6. Huang L, Damle SS, Booten S, et al. Partial hepatectomy induced long noncoding RNA inhibits hepatocyte proliferation during liver regeneration. PLOS ONE. 2015;10:e0132798.

7. Wu Y, Lyu H, Liu H, Shi X, Song Y, Liu B. Downregulation of the long noncoding RNA GAS5-AS1 contributes to tumor metastasis in nonsmall cell lung cancer. Sci Rep. 2016;6:31093.

8. Zhang A, Zhao JC, Kim J, et al. LncRNA HOTAIR enhances the androgen-receptor-mediated transcriptional program and drives castration-resistant prostate cancer. Cell Rep. 2015;13:209-221.

9. Mercer TR, Dinger ME, Mattick JS. Long non-coding RNAs: insights into functions. Nat Rev Genet. 2009;10:155-159.

10. Sasaki H, Ishihara K, Kato R. Mechanisms of Igf2/H19 imprinting: DNA methylation, chromatin and long-distance gene regulation. J Biochem. 2000;127:711-715.

11. Zhang J, Liu SC, Luo XH, et al. Exosomal long noncoding RNAs are differentially expressed in the cervicovaginal lavage samples of cervical cancer patients. J Clin Lab Anal. 2016;30:1116-1121.

12. Xia T, Chen S, Jiang Z, et al. Long noncoding RNA FER1L4 suppresses cancer cell growth by acting as a competing endogenous RNA and regulating PTEN expression. Sci Rep. 2015;5:13445.

13. Schmitt AM, Chang HY. Long noncoding RNAs in cancer pathways. Cancer Cell. 2016;29:452-463.

14. Graham LD, Pedersen SK, Brown GS, et al. Colorectal neoplasia differentially expressed (CRNDE), a novel gene with elevated expression in colorectal adenomas and adenocarcinomas. Genes Cancer. 2011;2:829-840

15. Ellis BC, Molloy PL, Graham LD. CRNDE: a long non-coding RNA involved in Cancer, neurobiology, and development. Front Genet. 2012;3:270.

16. Liu T, Zhang X, Yang YM, Du LT, Wang CX. Increased expression of the long noncoding RNA CRNDE-h indicates a poor prognosis in colorectal cancer, and is positively correlated with IRX5 mRNA expression. OncoTargets Ther. 2016;9:1437-1448.

17. Ding J, Li J, Wang H, et al. Long noncoding RNA CRNDE promotes colorectal cancer cell proliferation via epigenetically silencing DUSP5/ CDKN1A expression. Cell Death Dis. 2017;8:e2997.

18. Gao $\mathrm{H}$, Song $\mathrm{X}$, Kang $\mathrm{T}$, et al. Long noncoding RNA CRNDE functions as a competing endogenous RNA to promote metastasis and oxaliplatin resistance by sponging miR-136 in colorectal cancer. OncoTargets Ther. 2017;10:205-216.

19. Han P, Li JW, Zhang BM, et al. The IncRNA CRNDE promotes colorectal cancer cell proliferation and chemoresistance via miR-181a-5p-mediated regulation of Wnt/beta-catenin signaling. Mol Cancer. 2017;16:9.

20. Jiang $\mathrm{H}$, Wang $\mathrm{Y}, \mathrm{Ai} \mathrm{M}$, et al. Long noncoding RNA CRNDE stabilized by hnRNPUL2 accelerates cell proliferation and migration in 
colorectal carcinoma via activating Ras/MAPK signaling pathways. Cell Death Dis. 2017;8:e2862.

21. Yu B, Ye X, Du Q, Zhu B, Zhai Q, Li XX. The long non-coding RNA CRNDE promotes colorectal carcinoma progression by competitively binding miR-217 with TCF7L2 and enhancing the Wnt/beta-Catenin signaling pathway. Cell Physiol Biochem. 2017;41:2489-2502.

22. Zhang $X$, Sun $S, P u J K$, et al. Long non-coding RNA expression profiles predict clinical phenotypes in glioma. Neurobiol Dis. 2012;48:1-8.

23. Murat A, Migliavacca E, Gorlia T, et al. Stem cell-related "self-renewal" signature and high epidermal growth factor receptor expression associated with resistance to concomitant chemoradiotherapy in glioblastoma. J Clin Oncol. 2008;26:3015-3024.

24. Grzmil M, Morin P Jr, Lino MM, et al. MAP kinase-interacting kinase 1 regulates SMAD2-dependent TGF-beta signaling pathway in human glioblastoma. Can Res. 2011;71:2392-2402.

25. Chen $Y, W u J J$, Lin $X B$, et al. Differential IncRNA expression profiles in recurrent gliomas compared with primary gliomas identified by microarray analysis. Int J Clin Exp Med. 2015;8:5033-5043.

26. Chen Z, Yu C, Zhan L, Pan Y, Chen L, Sun C. LncRNA CRNDE promotes hepatic carcinoma cell proliferation, migration and invasion by suppressing miR-384. Am J Cancer Res. 2016;6:2299-2309.

27. Dong R, Liu XQ, Zhang BB, Liu BH, Zheng S, Dong KR. Long noncoding RNA-CRNDE: a novel regulator of tumor growth and angiogenesis in hepatoblastoma. Oncotarget. 2017;8:42087-42097.

28. Jin B, Wang W, Du G, et al. Identifying hub genes and dysregulated pathways in hepatocellular carcinoma. Eur Rev Med Pharmacol Sci. 2015;19:592-601.

29. Zhang $M$, Gao C, Yang Y, et al. Long noncoding RNA CRNDE/PRC2 participated in the radiotherapy resistance of human lung adenocarcinoma through targeting p21 expression. Oncol Res. 2017. https://doi. org/10.3727/096504017X14944585873668

30. Kiang KM, Zhang XQ, Zhang GP, et al. CRNDE expression positively correlates with EGFR activation and modulates glioma cell growth. Target Oncol. 2017;12:353-363.

31. Zheng J, Liu X, Wang P, et al. CRNDE promotes malignant progression of glioma by attenuating miR-384/PIWIL4/STAT3 Axis. Mol Ther. 2016;24:1199-1215.

32. Huan J, Xing L, Lin Q, Xui H, Qin X. Long noncoding RNA CRNDE activates Wnt/beta-catenin signaling pathway through acting as a molecular sponge of microRNA-136 in human breast cancer. Am J Trans Res. 2017;9:1977-1989.

33. Liu T, Zhang X, Gao S, et al. Exosomal long noncoding RNA CRNDE-h as a novel serum-based biomarker for diagnosis and prognosis of colorectal cancer. Oncotarget. 2016;7:85551-85563.

34. Yu B, Du Q, Li H, et al. Diagnostic potential of serum exosomal colorectal neoplasia differentially expressed long non-coding RNA (CRNDE-p) and microRNA-217 expression in colorectal carcinoma. Oncotarget. 2017;8:83745-83753.

35. Ellis BC, Graham LD, Molloy PL. CRNDE, a long non-coding RNA responsive to insulin/IGF signaling, regulates genes involved in central metabolism. Biochem Biophys Acta. 2014;1843:372-386.

36. Wang Y, Wang Y, Li J, Zhang Y, Yin H, Han B. CRNDE, a long-noncoding RNA, promotes glioma cell growth and invasion through mTOR signaling. Cancer Lett. 2015;367:122-128.

37. Jing SY, Lu YY, Yang JK, Deng WY, Zhou Q, Jiao BH. Expression of long non-coding RNA CRNDE in glioma and its correlation with tumor progression and patient survival. Eur Rev Med Pharmacol Sci. 2016;20:3992-3996.

38. Zheng J, Li XD, Wang P, et al. CRNDE affects the malignant biological characteristics of human glioma stem cells by negatively regulating miR-186. Oncotarget. 2015;6:25339-25355.

39. Kiang KMY, Leung GKK. Clinical significance of CRNDE transcript variants in glioblastoma multiforme. Non coding RNA Res. 2017;2:119-121.
40. Zhang XQ, Leung GK. Long non-coding RNAs in glioma: functional roles and clinical perspectives. Neurochem Int. 2014;77:78-85.

41. Li H, Li Q, Guo T, He W, Dong C, Wang Y. LncRNA CRNDE triggers inflammation through the TLR3-NF-kappaB-Cytokine signaling pathway. Tumour Biol. 2017;39:1-8.

42. Esposti DD, Hernandez-Vargas $\mathrm{H}$, Voegele $\mathrm{C}$, et al. Identification of novel long non-coding RNAs deregulated in hepatocellular carcinoma using RNA-sequencing. Oncotarget. 2016;7:31862-31877.

43. Liu XX, Xiong HP, Huang JS, Qi K, Xu JJ. Highly expressed long noncoding RNA CRNDE promotes cell proliferation through PI3K/AKT signalling in non-small cell lung carcinoma. Clin Exp Pharmacol Physiol. 2017;44:895-902.

44. Colvin EK, Vafaee F, Mok SC, Howell VM, Samimi G. Differential expression of long non-coding RNAs in ovarian cancer-associated fibroblasts versus normal ovarian fibroblasts. Can Res. 2015;75:S15.

45. Szafron LM, Balcerak A, Grzybowska EA, et al. The putative oncogene, CRNDE, is a negative prognostic factor in ovarian cancer patients. Oncotarget. 2015;6:43897-43910.

46. Szafron L, Balcerak A, Grzybowska EA, Rubel T, Kupryjanczyk J. The CRNDE, VAV2 and CEBPA genes as new negative prognostic factors in ovarian cancer patients. Eur J Cancer. 2013;49:S216-S216.

47. Han $\mathrm{Y}$, Lao M, Lei Q, et al. Expression and clinical significance of long non-coding RNA CRNDE in cervical cancer. Chin J Clin Oncol. 2015;42:705-708

48. Du J, Luo H, Xi H, Qu Y, Zhao Y, Hou J. Analysis of the gep dataset identifies Incrnas differential expression pattern and prognosis model of multiple myeloma. Clin Lymphoma Myeloma Leuk. 2013;13:S55.

49. Meng YB, He X, Huang YF, Wu QN, Zhou YC, Hao DJ. Long noncoding RNA CRNDE promotes multiple myeloma cell growth by Suppressing miR-451. Oncol Res. 2017;25:1207-1214.

50. Subhash S, Andersson PO, Kosalai ST, Kanduri C, Kanduri M. Global DNA methylation profiling reveals new insights into epigenetically deregulated protein coding and long noncoding RNAs in CLL. Clin Epigenetics. 2016;8:106

51. Maguire S, Leonidou A, Wai P, et al. SF3B1 K700E mutations are associated with alternative splicing in oestrogen receptor-positive breast cancer. Virchows Arch. 2014;465:S24.

52. Shao K, Shi T, Yang Y, Wang X, Xu D, Zhou P. Highly expressed IncRNA CRNDE promotes cell proliferation through Wnt/beta-catenin signaling in renal cell carcinoma. Tumour Biol. 2016;37:15997-16004.

53. Yang FY, Wang Y, Wu JG, et al. Analysis of long non-coding RNA expression profiles in clear cell renal cell carcinoma. Oncology Lett. 2017;14:2757-2764.

54. Hajjari M, Khoshnevisan A. Potential long non-coding RNAs to be considered as biomarkers or therapeutic targets in gastric cancer. Front Genetics. 2013;4:210.

55. Shen $\mathrm{S}$, Liu $\mathrm{H}$, Wang Y, et al. Long non-coding RNA CRNDE promotes gallbladder carcinoma carcinogenesis and as a scaffold of DMBT1 and C-IAP1 complexes to activating PI3K-AKT pathway. Oncotarget. 2016;7:72833-72844.

56. Lili LN, Matyunina LV, Walker LD, Daneker GW, McDonald JF. Evidence for the importance of personalized molecular profiling in pancreatic cancer. Pancreas. 2014;43:198-211.

57. Luo $\mathrm{YH}$, Liang $\mathrm{L}$, He RQ, et al. RNA-sequencing investigation identifies an effective risk score generated by three novel IncRNAs for the survival of papillary thyroid cancer patients. Oncotarget. 2017;26:26.

How to cite this article: Zhang J, Yin M, Peng G, Zhao Y. CRNDE: An important oncogenic long non-coding RNA in human cancers. Cell Prolif. 2018;51:e12440. https://doi. org/10.1111/cpr.12440 